\title{
Haptoglobin genotype and outcome after spontaneous intracerebral haemorrhage
}

Isabel C Hostettler MD*1, Matthew J Morton $\mathrm{PhD}^{* 2}$, Gareth Ambler $\mathrm{PhD}^{3}$, Nabila Kazmi ${ }^{4,5}$, Tom Gaunt ${ }^{4,5}$, Duncan Wilson $\mathrm{PhD}^{1,6}$, Clare Shakeshaft Msc ${ }^{1}$, Hans R Jäger $\mathrm{MD}^{7}$, Hannah Cohen $\mathrm{PhD}^{8}$, Tarek Yousry MD ${ }^{7}$, Rustam Al-Shahi Salman $\mathrm{PhD}^{9}$, Gregory Y H Lip FRCP ${ }^{10,11}$, Martin M Brown FRCP ${ }^{1}$, Keith W Muir MD FRCP ${ }^{12}$, Henry Houlden PhD $^{13}$, Diederik Bulters FRCS ${ }^{14}$, Ian Galea FRCP $\mathrm{PhD}^{2 \#}$, David J Werring FRCP PhD ${ }^{1 \#}$ on behalf of the CROMIS-2 collaborators

${ }^{1}$ Stroke Research Centre, University College London, Institute of Neurology, London, UK

${ }^{2}$ Clinical Neurosciences, Clinical and Experimental Sciences, Faculty of Medicine, University of Southampton, Southampton, UK

${ }^{3}$ Department of Statistical Science, UCL, London, WC1E 6BT, UK

${ }^{4}$ MRC Integrative Epidemiology Unit (IEU), Faculty of Health Sciences, University of Bristol, Bristol, UK

${ }^{5}$ Population Health Sciences, Bristol Medical School, University of Bristol, Bristol, UK

${ }^{6}$ New Zealand Brain Research Institute, Christchurch, New Zealand

${ }^{7}$ Neuroradiological Academic Unit, Department of Brain Repair \& Rehabilitation, University College London, Institute of Neurology, London, UK

${ }^{8}$ Haemostasis Research Unit, Department of Haematology, University College London, 51 Chenies Mews, London, UK

${ }^{9}$ Centre for Clinical Brain Sciences, School of Clinical Sciences, University of Edinburgh, Edinburgh, UK 
${ }^{10}$ Liverpool Centre for Cardiovascular Science, University of Liverpool and Liverpool Heart \& Chest Hospital, Liverpool, United Kingdom

${ }^{11}$ Aalborg Thrombosis Research Unit, Department of Clinical Medicine, Aalborg University, Aalborg, Denmark

${ }^{12}$ Institute of Neuroscience \& Psychology, University of Glasgow, Queen Elizabeth University Hospital, Glasgow, UK

${ }^{13}$ Neurogenetics Laboratory, The National Hospital of Neurology and Neurosurgery, London, $U K$

${ }^{14}$ Department of Neurosurgery, University Hospital Southampton NHS Foundation Trust, Southampton UK

\# joint senior authors

Corresponding author: Professor David Werring, FRCP, PhD, National Hospital of Neurology and Neurosurgery, Institute of Neurology, University College London, Queen Square, WC1N London, United Kingdom, Phone: +44 203447 5994, Fax: +44 207833 8613, Email: d.werring@ucl.ac.uk

Statistical analysis conducted by Isabel C Hostettler, MD and Gareth Ambler, PhD, University College London.

Word count: 3319/3500

Number of references: 40

Keywords: Intracerebral haemorrhage, Haptoglobin, intracerebral haemorrhage volume, oedema extension distance, perihaematomal oedema volume, functional outcome, death, ALSPAC

Sources of funding: DJW and DW received funding from the Stroke Foundation/British Heart Foundation. This work was undertaken at UCLH/UCL which receives a proportion of funding 
from the Department of Health’s National Institute for Health Research (NIHR) Biomedical Research Centers funding scheme. MM and IG received funding from the Medical Research Council (MR/L01453X/1). NK received funding from Cancer Research UK program grant C18281/A19169. The UK Medical Research Council (MRC) and Wellcome Trust (Grant ref: 102215/2/13/2) and the University of Bristol provide core support for ALSPAC. This publication is the work of the authors and ICH will serve as guarantor of the contents of this paper. A comprehensive list of grant funding is available on the ALSPAC website (http://www.bristol.ac.uk/alspac/external/documents/grant-acknowledgements.pdf).

\section{CONFLICT OF INTEREST}

The authors report no conflict of interest concerning the materials or methods used in this study or the findings specified in this paper.

\section{ACKNOWLEDGEMENTS}

We are extremely grateful to all patients, hospital staff and researcher who took part in this study. We also want to thank the whole ALSPAC team, which includes interviewers, computer and laboratory technicians, clerical workers, research scientists, volunteers, managers, receptionists and nurses. 


\section{CONTRIBUTORSHIP STATEMENT}

Isabel C Hostettler: Design and conceptualized study; Acquisition of data; performed laboratory work; analysed the data; drafted the manuscript; revised the manuscript Matthew J Morton: performed laboratory work; analysed the data; drafted the manuscript; revised the manuscript

Gareth Ambler: Design and conceptualized study; analysed the data; drafted the manuscript; revised the manuscript

Nabila Kazmi: analysed the data; drafted the manuscript; revised the manuscript

Tom Gaunt: analysed the data; drafted the manuscript; revised the manuscript

Duncan Wilson: Acquisition of data; analysed the data; drafted the manuscript; revised the manuscript

Clare Shakeshaft: Design and conceptualized study; Acquisition of data; revised the manuscript

Hans R Jäger: Design and conceptualized study; Acquisition of data; revised the manuscript

Hannah Cohen: Design and conceptualized study; of data; drafted the manuscript; revised the manuscript

Tarek Yousry: Design and conceptualized study; Design and conceptualized study; drafted the manuscript; revised the manuscript

Rustam Al-Shahi Salman: Design and conceptualized study; Acquisition of data; drafted the manuscript; revised the manuscript

Gregory Y H Lip: Design and conceptualized study; Acquisition of data; drafted the manuscript; revised the manuscript

Martin M Brown: Design and conceptualized study; Acquisition of data; drafted the manuscript; revised the manuscript

Keith W Muir: Design and conceptualized study; Acquisition of data; drafted the manuscript; revised the manuscript

Henry Houlden: Design and conceptualized study; Acquisition of data; drafted the manuscript; revised the manuscript

Diederik Bulters: Design and conceptualized study; Acquisition of data; analysed the data; drafted the manuscript; revised the manuscript

Ian Galea: Design and conceptualized study; Interpreted the data; revised the manuscript for intellectual content 
David J Werring: Design and conceptualized study; Interpreted the data; revised the manuscript for intellectual content; obtained funding for the study 


\section{ABSTRACT}

Objective: Haptoglobin is a haemoglobin-scavenging protein that binds and neutralises free haemoglobin and modulates inflammation and endothelial progenitor cell function. A HP gene copy number variation (CNV) generates HP1 and HP2 allele, the single nucleotide polymorphism rs2000999 influences their levels. HP1 allele is hypothesized to improve outcome after intracerebral haemorrhage (ICH). We investigated the associations of the HP CNV genotype and rs2000999 with haematoma volume, perihaematomal oedema (PHO) volume, and functional outcome as well as mortality after ICH.

Methods: We included patients with neuroimaging-proven ICH, available DNA, and six-month follow-up in an observational cohort study (CROMIS-2). We classified patients into three groups according to the HP CNV: $1-1,2-1$ or $2-2$ and also dichotomized HP into HP1containing genotypes (HP1-1 and HP2-1) and HP2-2 to evaluate the HP1 allele. We measured ICH and PHO volume on CT; PHO was measured by oedema extension distance. Functional outcome was assessed by modified Rankin score (unfavourable outcome defined as mRS 3-6). Results: We included 731 patients (mean age 73.4, 43.5\% female). Distribution of HP CNV genotype was: HP1-1 n=132 (18.1\%); HP2-1 n=342 (46.8\%); and HP2-2 n=257 (35.2\%). In the multivariable model mortality comparisons between HP groups, HP2-2 as reference, were as follows: OR HP1-1 0.73, 95\%CI 0.34-1.56 (p-value=0.41) and OR HP2-1 0.5, 95\%CI 0.280.89 (p-value=0.02) (overall p-value=0.06). We found no evidence of association of HP CNV or rs200999 with functional outcome, ICH volume or PHO volume.

Conclusion: The HP2-1 genotype might be associated with lower 6-month mortality after ICH; this finding merits further study. 


\section{INTRODUCTION}

Spontaneous (non-traumatic) intracerebral haemorrhage (ICH) is the most devastating form of stroke with a mortality of about $40 \%$ at one month, and $65 \%$ at one year ${ }^{1-3}$. Patients who survive frequently remain severely disabled ${ }^{4}$. Moreover, incidence of ICH is increasing in the elderly population $^{5-7}$, in part due to increasing use of oral anti-coagulation ${ }^{5-7}$.

Spontaneous ICH results from bleeding into the brain parenchyma arising from the rupture of an arterial vessel, most often (>80\%) a small arteriole affected by cerebral small vessel diseases (SVD). The commonest sporadic SVD that cause ICH are deep perforator arteriopathy (also termed hypertensive arteriopathy or arteriolosclerosis) and cerebral amyloid angiopathy (CAA). A minority of ICH (less than 20\%) is caused by structural or macrovascular bleeding sources such as tumours, arteriovenous malformations, cavernomas or fistulas. Deep perforator arteriopathy is associated with hypertension and is a frequent cause of deep ICH; CAA is caused by amyloid beta deposition in cortical and leptomeningeal blood vessels and is a key cause of lobar ICH.

Haptoglobin is an acute-phase protein which neutralizes free haemoglobin by binding it, and in doing so targets haemoglobin to the CD163 receptor for clearance ${ }^{8-15}$. Haptoglobin prevents the toxic and inflammatory effects of haemoglobin by shielding its iron-containing pocket, and preventing its breakdown into haem and iron, which consequently cause cytotoxicity and brain oedema $^{8-15}$. The HP gene has a copy number variant (CNV), which leads to two co-dominant alleles: HP1 and HP2. Three different HP CNV genotypes exist: HP1-1, HP2-1 and HP2-2, and their respective protein products differ in molecular size and haemoglobin-binding capacity $^{15-17}$. A previous study demonstrated some evidence that patients with the HP2 allele have a larger haematoma volume, though the underlying mechanisms remain unknown ${ }^{18}$. An increase in haematoma volume may be accompanied by more perihaematomal oedema $(\mathrm{PHO})^{18}{ }^{19}$. ICH and PHO volume have been demonstrated to influence functional outcome ${ }^{18}$ 
19. A previous study reported worse functional outcome for patients with HP2 allele (HP2-1 or 2-2) compared to HP1-1 patients as well as some evidence for increased mortality for each HP2 allele ${ }^{18}$. The HP CNV might be associated with functional outcome after ICH through differences in haemoglobin clearance and protection from the cytotoxic and inflammatory effects of haemoglobin breakdown products. However most previous studies investigating haptoglobin in ICH are based on investigations in rodents.

The single nucleotide polymorphism (SNP) rs2000999 accounts for up to 50\% of variation in circulating haptoglobin levels in the blood independently of the $H P \mathrm{CNV}^{20}$. The combined use of the HP CNV and rs2000999 has been suggested as an important genetic tool to discriminate between two potential mechanisms underlying differences between HP1 and HP2 alleles: haptoglobin expression level and functional differences in haptoglobin protein products ${ }^{21}$. We performed a comprehensible multivariable study investigating the influence of the $H P$ CNV and rs2000999 SNP on functional outcome and mortality after ICH. We also aimed to assess the influence of the HP CNV and the rs2000999 SNP on ICH volume and OED.

\section{METHODS}

\section{Data collection}

We considered patients, of predominantly Caucasian descent, with spontaneous ICH and available blood samples recruited into the Clinical Relevance of Microbleeds in Stroke ICH study $^{22}$. We defined spontaneous ICH as a non-traumatic haemorrhage into the brain parenchyma, presumed due to cerebral SVD after the exclusion of patients with an underlying structural or macrovascular cause.

We collected detailed information on demographics, risk factors, medication, clinical presentation, and radiological data. A diagnosis of hypertension, hypercholesterolaemia and diabetes mellitus was present if reported by the patient, stated on medical records or if either 
drug treatment or any other form of advice (including lifestyle changes) was given. Smoking was defined as current and previous use. All patients had acute brain imaging with CT. Written informed consent was obtained from all participants, or a relative or representative. We excluded patients $<18$ years, patients without available or adequate CT scan. Patients with a CT scan after 72 hours from symptom onset were excluded from the primary ICH and PHO volume analysis. ${ }^{18} 2324$. We classified ICH location into lobar, deep (basal ganglia, thalamus), cerebellar and brainstem according to a validated rating scale ${ }^{25}$. Our outcomes were death and functional outcome at 6 months (measured by the modified Rankin Scale [mRS] dichotomized into favorable [mRS 0-2] or unfavorable [mRS 3-6] categories).

\section{Haptoglobin genotyping}

To determine the HP CNV we optimised a high-throughput qPCR genotyping assay as described previously ${ }^{26}$. The assay amplified a region in the $5 `$ terminal of the HP gene’s first exon as an internal control (HP5`), and the breakpoint of the HP duplication (HP2). The HP2/HP5 ratio (theoretically either 0,1 , or 2) was used to determine the genotype as HP1-1, HP2-1 or HP2-2 respectively. Samples were run in triplicates; triplicates with a HP2/HP5 `ratio coefficient of variation $>10 \%$ were re-assayed. A second method of $H P$ genotyping by $\mathrm{PCR}^{27}$ was performed on samples with HP2/HP5' ratio values between 0.46-077, in order to confirm the HP CNV genotype. Rs2000999 was genotyped using Kompetitive Allele Specific PCR (KASP) assay technology ${ }^{28}$ (LGC Genomics Limited, Hertfordshire, UK), call rate was 97.3\%.

\section{Measurement of ICH and PHO volume}

We measured ICH and PHO volume as previously described via a semi-automated, thresholdbased approach $^{29}$. PHO was measured by the oedema extension distance (OED) using a previously described formula ${ }^{19}$; the rationale behind using OED is that PHO extends a consistent mean linear distance from the border of the ICH, independently of its volume. 


\section{Statistical analysis}

We present categorical variables using frequency and percentages, continuous variables using mean \pm standard deviation (SD). We transformed ICH and PHO volume with cube root transformation to satisfy statistical normal distribution assumptions. We conducted a post hoc sensitivity analysis comparing patients with ICH volume and OED before and after 72 hours. We assessed the distribution of the HP CNV and rs2000999 SNP in the CROMIS-2 cohort compared to ALSPAC (Avon Longitudinal Study of Parents and Children) cohort of healthy individuals, which we used as controls. ALSPAC is a general population cohort study ${ }^{30}{ }^{31}$; HP genetic data and rs2000999 SNP data was available from 927 and 748 participants. The ALSPAC study website (http://www.bristol.ac.uk/alspac/researchers/our-data/) contains details of all the data available through a fully searchable data dictionary and variable search tool. Ethical approval for the study was obtained from the ALSPAC Ethics and Law Committee and the Local Research Ethics Committees. To evaluate the HP1 allele, we also assessed the HP CNV as a dichotomized variable (HP1-1 and HP2-1 versus HP2-2) according to our prespecified analysis plan.

We first performed univariable analyses for each of the four outcomes separately with demographic, clinical and radiological variables of interest. We subsequently fitted multivariable logistic regression models with significant variables from the univariable analysis in addition to pre-specified variables. For the analysis of ICH and OED volume we adjusted the models with the pre-specified variables: time from event to imaging, location of ICH, systolic blood pressure (SBP), HP CNV and rs200999 SNP. For functional outcome and mortality analysis, we fitted the multivariable model with the pre-specified variables: age, sex, hypertension, oral anticoagulation (OAC), HP CNV and rs200999 SNP. Additionally, we fitted the multivariable models with variables that were statistically significant at the $20 \%$ level in the univariable analysis. 
We investigated whether there were interactions between different variables. However, no interaction reached our pre-specified significant threshold for interactions of $\mathrm{p}<0.001$ (chosen to guard against overfitting) and were therefore not included in the models ${ }^{32}$.

Statistical analysis was performed using STATA 15 (StataCorp. 2011. Stata Statistical Software: Release 15. College Station, TX: StataCorp LP).

\section{Ethical approval}

The CROMIS-2 study was approved by the local Ethics Committee (reference: 10/H0716/64).

\section{RESULTS}

For the primary analysis of functional outcome at 6 months we included 732 patients. One DNA sample was uncallable for the HP CNV and 20 for the rs2000999 SNP. For the secondary analyses of ICH volume and PHO we included 709 patients with an available CT scan (Figure 1). OED mas measured at a mean of 10 hours from ICH onset. Patients who were genotyped $(n=844)$ were not different to those without DNA $(n=250)$ with regard to baseline characteristics and risk factor profile (data not shown). The rs2000999 genotype frequency in CROMIS-2 was as expected when compared to ALSPAC (Supplementary Table 1). However, compared to ALSPAC, CROMIS-2 patients less often had the HP2-2 CNV. We found no systematic difference in demographics, comorbidities and ICH characteristics between those with and without available outcome variable (data not shown).

\section{Mortality}

Of 731 patients with available follow-up and genotype data, 112 died within 6 months (15.3\%) and 318 (43.5\%) were female. 
The distribution of the HP CNV was 132 HP1-1 (18.1\%), 342 HP2-1 (46.8\%) and 257 HP2-2 (35.2\%). Distribution of the SNP allele was: 27 A:A (3.8\%), 234 A:G (32.9\%) and 451 G:G (63.3\%), 20 samples were not callable (2.7\%).

Patients who died were older, more frequently female, more frequently on OAC, had a lower GCS on admission (GCS <8), a higher ICH and PHO volume, and intraventricular extension (IV). Results of the univariable analysis are shown in supplementary Table 2.

The mortality according to HP CNV was as follows: HP1-1 18.2\%; HP2-1 12.6\%; HP2-2 17.5\%. In the multivariable model $(\mathrm{n}=608)$ mortality comparisons between the HP groups, with HP2-2 as a reference group, were as follows: OR HP1-1 0.73, 95\% CI 0.34-1.56 (pvalue $=0.41)$ and OR HP2-1 0.5, 95\% CI 0.28-0.89 (p-value=0.02) (overall p-value=0.06, Table $1)$. 
Table 1: Factors associated with 6 month mortality after ICH in an adjusted multivariable logistic regression model

\begin{tabular}{|c|c|c|c|}
\hline & OR & $95 \% \mathrm{CI}$ & $P$ value \\
\hline Age (years) & 1.11 & $1.07-1.14$ & $<0.001$ \\
\hline Female Sex & 1.14 & $0.68-1.92$ & 0.63 \\
\hline Hypertension & 1.01 & $0.57-1.76$ & 0.99 \\
\hline Diabetes mellitus & 1.31 & $0.65-2.65$ & 0.46 \\
\hline Oral anticoagulation & 1.25 & $0.74-2.11$ & 0.4 \\
\hline $\begin{array}{l}\text { GCS on admission (bine } \\
\text { - GCS 3-8 } \\
\text { - GCS 9-15 (reference) }\end{array}$ & 4.23 & $1.35-13.28$ & 0.01 \\
\hline $\begin{array}{l}\text { ICH location } \\
\text { - Cerebellar (reference) } \\
\text { - Brainstem } \\
\text { - Deep } \\
\text { - Lobar }\end{array}$ & $\begin{array}{c}\text { Empty } \\
0.98 \\
0.64\end{array}$ & $\begin{array}{c}0.33-2.93 \\
0.2-2\end{array}$ & 0.38 \\
\hline $\mathrm{Cr}$ ICH volume (mL) & 2.03 & $1.48-2.8$ & $<0.001$ \\
\hline $\mathrm{OED}(\mathrm{cm})$ & 2.82 & $1.01-7.92$ & 0.05 \\
\hline IV extension & 1.56 & $0.89-2.72$ & 0.12 \\
\hline HP CNV & & & 0.06 \\
\hline $\begin{array}{l}\text { - HP1-1 } \\
\text { - HP2-1 } \\
\text { - HP2-2 (reference) }\end{array}$ & $\begin{array}{c}0.73 \\
0.5\end{array}$ & $\begin{array}{l}0.34-1.56 \\
0.28-0.89\end{array}$ & \\
\hline $\begin{array}{l}\text { Rs2000999 } \\
\text { - A:A (reference) } \\
\text { - A:G } \\
\text { - G:G }\end{array}$ & $\begin{array}{c}0.6 \\
0.58\end{array}$ & $\begin{array}{l}0.15-2.36 \\
0.15-2.28\end{array}$ & 0.74 \\
\hline
\end{tabular}

$\mathrm{cm}=$ centimeter; $\mathrm{CNV}=$ copy number variation; $\mathrm{Cr}=$ cube root $; \mathrm{CT}=$ computed tomography; GCS = Glasgow Coma Scale; $H P=$ Haptoglobin; $\mathrm{ICH}=$ intracerebral haemorrhage; IV = intraventricular; $\mathrm{ml}=$ milliliter; OAC: oral anticoagulation; SBP: systolic blood pressure 
When dichotomizing HP into HP1-1/2-1 versus HP2-2 there was evidence for association of decreased mortality with the HP1 allele compared to HP2-2 (OR 0.55, 95\%CI 0.31-0.95, $\mathrm{p}=0.03$, supplementary Table 3). As expected, there was also evidence for an increase in mortality with increasing age (OR 1.11, 95\%CI 1.07-1.14, $\mathrm{p}<0.001$ ), decreased GCS on admission <9 (OR 4.37, 95\%CI 1.39-13.73, p=0.01), and ICH volume (OR 1.99, 95\%CI 1.452.74, $\mathrm{p}<0.001)$.

We further investigated the association between mortality and HP CNV across tertiles of all the covariates included in the multivariable model as a post hoc analysis. Mortality differed between the HP groups for older patients ( $>80$ years) with lower $(<12.2 \mathrm{~mL})$ ICH volume: in this subgroup, mortality was $26 \%$ for HP1-1, 14\% for HP2-1 and 42\% for HP2-2. Patients died at a median of 3.8 months after ICH. There was no difference (early vs. late death) in the time of death after ICH across HP CNV or rs2000999 groups, in the overall cohort or the subgroup of $>80$ years and $<12.2 \mathrm{~mL}$ ICH volume (regression data not shown, supplementary Figure 1 ). The mortality rate was similar across the HP groups for the remaining patients: $15 \%$ for HP11, 12\% for HP2-1 and 12\% for HP2-2. The association between mortality and HP CNV was confirmed across tertiles of all the other covariates. Finally, we investigated covariates not included in the multivariable model, to see whether they differed across HP genotypes, but found no bias to explain the association between mortality and HP CNV (data not shown).

\section{Functional outcome}

Of 731 patients, 444 (60.7\%) suffered an unfavourable outcome (mRS 3-6). Dichotomized unfavourable mRS according to HP CNV was as follows: HP1-1 64.4\%; HP2-1 59.7\%; HP2$260.3 \%$. 
Patients with an unfavourable outcome were older, more frequently female, on OAC, more frequently had hypertension, hypercholesterolaemia, presented with a lower GCS (GCS of 38), had a higher ICH and PHO volume and IV extension. See supplementary Table 2 for univariable analysis.

In the multivariable model ( $\mathrm{n}=623)$ age (OR 1.04, 1.02-1.06 95\%CI; $\mathrm{p}<0.001)$, female sex (OR 2.31; 1.58-3.37; 95\%CI; $\mathrm{p}<0.001)$ and the cube root of the ICH volume (OR 1.5; 1.22-1.85 95\%CI; $\mathrm{p}<0.001$ ) were significantly associated with functional outcome (Table 2). Neither HP CNV nor rs2000999 SNP were associated with functional outcome. 
Table 2: Factors associated with unfavourable outcome after ICH in an adjusted multivariable regression model

\begin{tabular}{|c|c|c|c|}
\hline & OR & $95 \% \mathrm{CI}$ & P value \\
\hline Age (years) & 1.04 & $1.02-1.06$ & $<0.001$ \\
\hline Female Sex & 2.31 & $1.58-3.37$ & $<0.001$ \\
\hline Hypertension & 1.37 & $0.92-2.04$ & 0.12 \\
\hline Diabetes mellitus & 1.18 & $0.71-1.97$ & 0.52 \\
\hline Oral anticoagulation & 1.16 & $0.77-1.73$ & 0.49 \\
\hline Antiplatelets & 1.08 & $0.7-1.69$ & 0.72 \\
\hline Hypercholesterolaemia & 1.17 & $0.78-1.75$ & 0.44 \\
\hline $\begin{array}{l}\text { GCS on admission (bina } \\
\text { - GCS 3-8 } \\
\text { - GCS 9-15 (reference) }\end{array}$ & 3.56 & $0.76-16.5$ & 0.11 \\
\hline Cr ICH volume (mL) & 1.5 & $1.22-1.85$ & $<0.001$ \\
\hline IV extension & 1.38 & $0.9-2.12$ & 0.14 \\
\hline Surgical evacuation & 1.84 & $0.45-7.5$ & 0.39 \\
\hline $\begin{array}{l}\text { HP CNV } \\
\text { - HP1-1 } \\
\text { - HP2-1 } \\
\text { - HP2-2 (reference) }\end{array}$ & $\begin{array}{l}1.17 \\
0.97\end{array}$ & $\begin{array}{l}0.67-2.03 \\
0.65-1.45\end{array}$ & 0.78 \\
\hline $\begin{array}{l}\text { Rs2000999 } \\
\text { - A:A (reference) } \\
\text { - A:G } \\
\text { - G:G }\end{array}$ & $\begin{array}{l}1.19 \\
1.39\end{array}$ & $\begin{array}{l}0.43-3.3 \\
0.5-3.84\end{array}$ & 0.66 \\
\hline
\end{tabular}

$\mathrm{CNV}=$ copy number variant; $\mathrm{Cr}=$ cube root CT = computed tomography; GCS = Glasgow Coma Scale; HP = Haptoglobin; ICH = intracerebral haemorrhage; IV = intraventricular; $\mathrm{ml}$ = millilitre; OAC: oral anticoagulation; SBP: systolic blood pressure 


\section{Intracerebral haemorrhage volume and oedema extension distance}

Of the 731 patients included in the functional analysis, 709 had a CT scan available, and of these 68 were $>72$ hours after symptom onset (Figure 1). Of the remaining 641 individuals, 453 (70.7\%) had a scan <24h, 172 (26.8\%) between 24-48h and 16 (2.5\%) between 48-72h.

See Figure 2 for the association of the HP CNV and SNP with OED and ICH volume.

Mean ICH volume was $13.8 \mathrm{~mL}$ ( \pm 18.82 SD), mean PHO volume $19.54 \mathrm{~mL}( \pm 20.56 \mathrm{SD})$ and mean OED $0.51 \mathrm{~cm}( \pm 0.23 \mathrm{SD})$. Variables significantly associated with $\mathrm{ICH}$ volume in the univariable analysis are listed in the supplementary Table 3.

In the fitted multivariable model $(\mathrm{n}=604)$ ICH location (overall $\mathrm{p}<0.001)$ and intraventricular extension (coefficient 0.53 ; 0.37-0.68; $\mathrm{p}<0.001$ ) were associated with greater ICH volume (Table 3). Neither HP CNV nor the SNP rs2000999 were associated with ICH volume. 
Table 3: Factors associated with the cube root ICH volume in an adjusted multivariable regression model

\section{Coefficient}

$95 \%$ CI

P value

Age (years)

$-0.005$

$-0.01-0.001$

0.09

Time Event to CT

0.35

- Day 1 (reference)

- Day 2

- Day 3

0.04

$-0.29$

$-0.23-0.31$

$-0.7-0.11$

ICH location

- Cerebellar (reference)

- Brainstem

$-0.73$

$-0.13$

0.79

$-1.22-0.23$

- Deep

$-0.44-0.18$

- Lobar

0.001

0.47-1.1

SBP (mmHg)

0.001

$-0.002-0.002$

0.88

Platelet level (x109/liter)

0.09

$-0.0004-0.001$

0.31

Hypercholesterolaemia

0.53

$-0.05-0.22$

0.2

IV extension

0.36

$0.37-0.68$

$<0.001$

Neurosurgery

$-0.06-0.78$

0.1

HP CNV

$-0.09$

$-0.02$

$-0.25-0.52$

- HP1-1

$-0.17-0.13$

- HP2-2 (reference)

Rs2000999

0.68

- A:A (reference)

- A:G

0.14

$-0.25-0.52$

- G:G

0.16

$-0.22-0.54$

$\mathrm{CNV}=$ copy number variation; $\mathrm{CT}=$ computed tomography; $\mathrm{HP}=$ Haptoglobin; $\mathrm{ICH}=$ intracerebral haemorrhage; IV= intraventricular; $\mathrm{mmHg}=$ millimetre mercury; $\mathrm{SBP}=$ systolic blood pressure 
After dichotomizing the HP CNV into HP1-1/2-1 versus HP2-2 we did not observe any evidence of an association in univariable or multivariable analyses ( $p=0.39$ [supplementary Table 4] and $\mathrm{p}=0.6$ respectively [data not shown]). Similar results were observed when dichotomizing HP CNV into HP1-1 versus HP2-1/2-2 [supplementary Table 4].

\section{Oedema Extension Distance}

Variables significantly associated with OED in the univariable analysis are listed in supplementary Table 4. For comparison of HP CNV and SNP for ICH volume and OED see Figure 2.

In the multivariable linear regression model ( $n=623)$, ICH location (with lobar and deep ICH locations featuring a longer OED and with a brainstem location featuring a shorter OED, compared to the reference group of cerebellar location, overall $\mathrm{p}<0.001$ ) and antihypertensive medication (coefficient -0.09; 95\%CI -0.16-(-0.02); $\mathrm{p}=0.01$ ) were significantly associated with OED (Table 4). Neither the univariable nor multivariable analysis showed evidence of association of HP CNV or rs2000999 SNP with OED.

Similar to the ICH volume model, dichotomizing HP did not yield any evidence of association in univariable and multivariable models (data not shown). 
Table 4: Factors associated with size of oedema extension distance in an adjusted multivariable regression model

$\begin{array}{lll}\text { Coefficient } & 95 \% \mathrm{CI} & \boldsymbol{P} \text { value }\end{array}$

Female Sex

0.01

$-0.02-0.05$

0.44

Time Event to CT

0.18

- Day 1 (reference)

- Day 2

- Day 3

$\begin{array}{ll}0.07 & -0.008-0.14 \\ 0.04 & -0.07-0.15\end{array}$

ICH location

- Cerebellar (reference)

- Brainstem

$\begin{array}{ll}-0.08 & -0.21-0.06 \\ 0.16 & 0.07-0.24 \\ 0.24 & 0.15-0.33\end{array}$

- Deep

0.24

0.49

SBP (mmHg)

0.0002

$-0.0003-0.001$

0.17

OAC

0.05

$-0.02-0.12$

Antihypertensive medication

$-0.09$

$-0.16-(-0.02)$

0.01

Platelet level (x109//iter)

$0.0002-0.00005-0.0004$

0.11

IV extension

$-0.03$

$-0.07-0.008$

0.11

HP CNV

- HP1-1

0.03

$-0.02-0.09$

0.01

$-0.03-0.05$

- HP2-2 (reference)

Rs2000999

- A:A (reference)

- A:G

0.01

$-0.09-0.11$

- G:G

0.003

$-0.1-0.1$

$\mathrm{CNV}=$ copy number variation; $\mathrm{CT}=$ computed tomography; $\mathrm{HP}=$ Haptoglobin; $\mathrm{ICH}=$ intracerebral haemorrhage; $\mathrm{mmHg}=$ millimetre mercury; OAC: oral anticoagulation; SBP: systolic blood pressure 


\section{DISCUSSION}

In this large prospective, multicentre cohort study, $H P$ was not associated with functional outcome as assessed by the mRS. The HP CNV distribution was comparable to that reported in a previous study, apart from a slightly higher proportion of HP1-1 patients and lower proportion of HP2- $2^{18}$. Despite the larger sample size, we could not replicate this previous study's finding of an association of the HP2 allele with functional outcome ${ }^{18}$.

However, we found evidence that mortality was lower in HP2-1 patients compared to HP2-2 homozygotes; our post hoc analyses suggest that this observation is mostly driven by older patients with lower ICH volumes. No association with mortality was found for the rs2000999 SNP (which is associated with haptoglobin expression level) ${ }^{21}$. This suggests that any link between the HP CNV and mortality is mediated by factors other than haptoglobin expression.

While the HP CNV's association with mortality could have been confounded by bias in a variable excluded from the model, we did not find any evidence for this. Such a factor could still remain unidentified, but a more likely explanation is that patients who died did not contribute to functional outcome analysis. We found evidence of HP2-2 missingness (of subjects of a particular genotype, in this case HP2-2), when comparing CROMIS-2 with ALSPAC cohorts, which might suggest that the HP2-2 genotype confers a mortality risk.

We confirmed previous results showing evidence towards increased mortality with HP2- ${ }^{18}$, but did not observe a unidirectional dose response of HP alleles in a direction of increasing or decreasing mortality across HP genotypes (mortality: HP1-1 18.2\%; HP2-1 12.6\%; HP2-2 17.5\%). The lower mortality in HP2-1 individuals could be a chance finding. A possible but unlikely explanation is heterozygote advantage or heterosis ${ }^{33}$. At a molecular level, the HP1 
allele might protect against the deleterious effect of the HP2 allele only when the two alleles are present together in HP2-1 individuals. Both HP1 and HP2 alleles scavenge haemoglobin, with HP2 being superior ${ }^{34} 35$, and this confers a beneficial effect. However, HP2 has additional off-target effects which are deleterious, mostly pro-inflammatory ${ }^{36}$. In HP2-2 individuals, the better haemoglobin scavenging potential of HP2 versus HP1 is offset by its proinflammatory effects, so that mortality is similar in HP1-1 and HP2-2 individuals. In HP2-1 individuals, the HP1 allele may be negating the deleterious effect of HP2, so that a greater benefit is observed in HP2-1 individuals than is expected by simple co-dominance of the two alleles.

We did not confirm previous findings of worse functional outcome in patients with HP2 allele, which could be due to the significantly smaller cohort size and statistical power of the previous study, with potential for a chance finding ${ }^{18}$.

PHO develops over a continuous period of time in three main stages. It peaks after two weeks, however its evolution is most rapid in the first 2-3 days ${ }^{37}$. PHO is thought to be mediated by a process of toxicity and inflammation ${ }^{19}{ }^{37}$. We hypothesized that by modulating neurotoxicity and inflammatory processes haptoglobin might have influenced $\mathrm{PHO}$ and functional outcome. ${ }^{38}$ However, we did not find any association of HP genetic variants (CNV or the rs2000999 SNP) with OED. Similarly, HP genetic variants were not associated with ICH volume, which, like haemtoma expansion, is more likely to be driven by other factors including hydrostatic pressure at the bleeding point ${ }^{18}$.

Despite having a large cohort available, we could not replicate the previous study's reported finding of an association of the HP2 allele with larger ICH volumes and IV extension ${ }^{18}$. Since ICH volume and OED was assessed on CT scans performed within 72 hours of symptom onset, 
we cannot exclude an association of $\mathrm{HP}$ with ICH volume or OED after this timepoint, although our exploratory analysis of scans beyond 72 hours $(n=68)$ and found no difference in ICH volume and OED across HP genotypes (for both CNV and rs2000999 SNP) (data not shown). We found that long-term antihypertensive medication prior to ICH event is independently associated with decreased OED, even after correcting for SBP. It is possible that patients on antihypertensive medication could have reduced sympathetic activity and inflammatory response when $\mathrm{ICH}$ occurs $^{39}$, a hypothesis that merits further study. As we did not collect follow-up scans, we cannot comment on a potential influence of SBP on haematoma growth.

Our study has strengths. Our prospective, multi-centre study is the largest on $\mathrm{HP}$ and ICH to date, and should be generalizable to Caucasian populations. We collected detailed baseline clinical and brain imaging data and undertook multivariable regression analysis adjusting and correcting for important predictors of all four outcomes, and took exceptional care to control for covariates.

However, our study also has limitations. Since we obtained informed or proxy consent, our study is biased towards ICH survivors with less severe ICH than would be included in an unselected incident ICH population. However, it is likely that any protective effect of HP is most relevant in ICH patients who survive the acute period. Additionally, CT scans at multiple timepoints were not available and therefore we could not assess the influence of HP CNV and rs200999 SNP on ICH, PHO or OED expansion over time. We also did not have data on the time interval between the ICH and CT scan. However, in a post hoc sensitivity analysis ICH volume before and after 72 hours was very similar although OED was larger in patients with first imaging after 72 hours. As PHO increases beyond 72 hours further studies are needed to assess an influence of the HP CNV and rs2000999 SNP on oedema expansion. Although we 
excluded patients without blood samples available for genetic analysis, there were no systematic differences in demographics, comorbidities and ICH characteristics between those with and without genetic data available. Finally, it would have been interesting to study plasma and cerebrospinal fluid haptoglobin levels in relation to HP genetic variants, but unfortunately these were not available.

\section{CONCLUSION}

We investigated the association of HP genetic variation (the HP CNV and the rs2000999 SNP) in a large cohort of 731 ICH patients. We found evidence in support of a lower mortality with the HP2-1 genotype, but not functional outcome, ICH volume or OED. While HP genotype may not matter for functional outcome, upregulating or supplementing haptoglobin may still be of benefit, as demonstrated in animal studies ${ }^{40}$, so understanding how different haptoglobin types associate with outcome is important. A future meta-analysis may be appropriate to confirm our observations, and longer follow-up may be needed in case there is an association with longer term outcome. 


\section{REFERENCES}

1. Bamford J, Sandercock P, Dennis M, et al. A prospective study of acute cerebrovascular disease in the community: the Oxfordshire Community Stroke Project--1981-86. 2. Incidence, case fatality rates and overall outcome at one year of cerebral infarction, primary intracerebral and subarachnoid haemorrhage. J Neurol Neurosurg Psychiatry 1990;53(1):16-22.

2. Poon MT, Fonville AF, Al-Shahi Salman R. Long-term prognosis after intracerebral haemorrhage: systematic review and meta-analysis. Journal of neurology, neurosurgery, and psychiatry 2014;85(6):660-7. doi: 10.1136/jnnp-2013-306476

3. van Asch CJ, Luitse MJ, Rinkel GJ, et al. Incidence, case fatality, and functional outcome of intracerebral haemorrhage over time, according to age, sex, and ethnic origin: a systematic review and meta-analysis. The Lancet Neurology 2010;9(2):167-76. doi: 10.1016/S1474-4422(09)70340-0 [published Online First: 2010/01/09]

4. Sudlow CL, Warlow CP. Comparable studies of the incidence of stroke and its pathological types: results from an international collaboration. International Stroke Incidence Collaboration. Stroke; a journal of cerebral circulation 1997;28(3):491-9.

5. Bejot $Y$, Cordonnier C, Durier J, et al. Intracerebral haemorrhage profiles are changing: results from the Dijon population-based study. Brain 2013;136(Pt 2):658-64. doi: 10.1093/brain/aws349 [published Online First: 2013/02/05]

6. Flaherty ML, Kissela B, Woo D, et al. The increasing incidence of anticoagulant-associated intracerebral hemorrhage. Neurology 2007;68(2):116-21. doi: 10.1212/01.wnl.0000250340.05202.8b

7. Lovelock CE, Molyneux AJ, Rothwell PM, et al. Change in incidence and aetiology of intracerebral haemorrhage in Oxfordshire, UK, between 1981 and 2006: a populationbased study. Lancet Neurol 2007;6(6):487-93. doi: 10.1016/S1474-4422(07)70107-2 [published Online First: 2007/05/19]

8. Huang FP, Xi G, Keep RF, et al. Brain edema after experimental intracerebral hemorrhage: role of hemoglobin degradation products. Journal of neurosurgery 2002;96(2):287-93. doi: 10.3171/jns.2002.96.2.0287 [published Online First: 2002/02/13]

9. Thiex R, Tsirka SE. Brain edema after intracerebral hemorrhage: mechanisms, treatment options, management strategies, and operative indications. Neurosurg Focus 2007;22(5):E6. [published Online First: 2007/07/07]

10. Xi G, Keep RF, Hoff JT. Erythrocytes and delayed brain edema formation following intracerebral hemorrhage in rats. Journal of neurosurgery 1998;89(6):991-6. doi: 10.3171/jns.1998.89.6.0991 [published Online First: 1998/12/02]

11. Andersen $C B$, Torvund-Jensen $M$, Nielsen MJ, et al. Structure of the haptoglobinhaemoglobin complex. Nature 2012;489(7416):456-9. doi: 10.1038/nature11369

12. Banerjee $\mathrm{S}$, Jia $\mathrm{Y}$, Siburt $\mathrm{CJ}$, et al. Haptoglobin alters oxygenation and oxidation of hemoglobin and decreases propagation of peroxide-induced oxidative reactions. Free radical biology \& medicine 2012;53(6):1317-26. doi: 10.1016/j.freeradbiomed.2012.07.023

13. Cooper CE, Schaer DJ, Buehler PW, et al. Haptoglobin binding stabilizes hemoglobin ferryl iron and the globin radical on tyrosine beta145. Antioxidants \& redox signaling 2013;18(17):2264-73. doi: 10.1089/ars.2012.4547 
14. Schaer CA, Vallelian F, Imhof A, et al. CD163-expressing monocytes constitute an endotoxin-sensitive $\mathrm{Hb}$ clearance compartment within the vascular system. Journal of leukocyte biology 2007;82(1):106-10. doi: 10.1189/jlb.0706453

15. Bulters D, Gaastra B, Zolnourian A, et al. Haemoglobin scavenging in intracranial bleeding: biology and clinical implications. Nature reviews Neurology 2018 doi: 10.1038/s41582018-0020-0 [published Online First: 2018/06/22]

16. Asleh R, Marsh S, Shilkrut M, et al. Genetically determined heterogeneity in hemoglobin scavenging and susceptibility to diabetic cardiovascular disease. Circulation research 2003;92(11):1193-200. doi: 10.1161/01.RES.0000076889.23082.F1

17. Langlois MR, Delanghe JR. Biological and clinical significance of haptoglobin polymorphism in humans. Clinical chemistry 1996;42(10):1589-600.

18. Murthy SB, Levy AP, Duckworth J, et al. Presence of haptoglobin-2 allele is associated with worse functional outcomes after spontaneous intracerebral hemorrhage. World Neurosurg 2015;83(4):583-7. doi: 10.1016/j.wneu.2014.12.013

19. Parry-Jones AR, Wang $X$, Sato $S$, et al. Edema Extension Distance: Outcome Measure for Phase II Clinical Trials Targeting Edema After Intracerebral Hemorrhage. Stroke; a journal of cerebral circulation 2015;46(6):e137-40. doi: 10.1161/STROKEAHA.115.008818 [published Online First: 2015/05/07]

20. Froguel $P$, Ndiaye NC, Bonnefond $A$, et al. A genome-wide association study identifies rs2000999 as a strong genetic determinant of circulating haptoglobin levels. PloS one 2012;7(3):e32327. doi: 10.1371/journal.pone.0032327

21. Kazmi N, Koda Y, Ndiaye NC, et al. Genetic determinants of circulating haptoglobin concentration. Clinica chimica acta; international journal of clinical chemistry 2019;494:138-42. doi: 10.1016/j.cca.2019.03.1617 [published Online First: 2019/03/23]

22. Charidimou A, Wilson D, Shakeshaft C, et al. The Clinical Relevance of Microbleeds in Stroke study (CROMIS-2): rationale, design, and methods. International journal of stroke : official journal of the International Stroke Society 2015;10 Suppl A100:155-61. doi: 10.1111/ijs.12569

23. Murthy SB, Urday S, Beslow LA, et al. Rate of perihaematomal oedema expansion is associated with poor clinical outcomes in intracerebral haemorrhage. Journal of neurology, neurosurgery, and psychiatry 2016;87(11):1169-73. doi: 10.1136/jnnp2016-313653

24. Urday S, Kimberly WT, Beslow LA, et al. Targeting secondary injury in intracerebral haemorrhage--perihaematomal oedema. Nature reviews Neurology 2015;11(2):11122. doi: $10.1038 /$ nrneurol.2014.264

25. Charidimou A, Schmitt A, Wilson D, et al. The Cerebral Haemorrhage Anatomical RaTing inStrument (CHARTS): Development and assessment of reliability. J Neurol Sci 2017;372:178-83. doi: 10.1016/j.jns.2016.11.021 [published Online First: 2016/12/27]

26. Soejima M, Koda Y. TaqMan-based real-time PCR for genotyping common polymorphisms of haptoglobin (HP1 and HP2). Clinical chemistry 2008;54(11):1908-13. doi: 10.1373/clinchem.2008.113126

27. Koch W, Latz W, Eichinger M, et al. Genotyping of the common haptoglobin $\mathrm{Hp} 1 / 2$ polymorphism based on PCR. Clinical chemistry 2002;48(9):1377-82.

28. Semagn K, Babu, R., Hearne, S., and Olsen, M. Single nucleotide polymorphism genotyping using Kompetitive Allele Specific PCR (KASP): overview of the technology and its 
application in crop improvement. Molecular Breeding 2014(33):1-14. doi: doi: 10.1007/s11032-013-9917-x

29. Volbers B, Staykov D, Wagner I, et al. Semi-automatic volumetric assessment of perihemorrhagic edema with computed tomography. European journal of neurology 2011;18(11):1323-8. doi: 10.1111/j.1468-1331.2011.03395.x [published Online First: 2011/04/05]

30. Boyd A, Golding J, Macleod J, et al. Cohort Profile: the 'children of the 90s'--the index offspring of the Avon Longitudinal Study of Parents and Children. International journal of epidemiology 2013;42(1):111-27. doi: 10.1093/ije/dys064 [published Online First: 2012/04/18]

31. Fraser A, Macdonald-Wallis C, Tilling K, et al. Cohort Profile: the Avon Longitudinal Study of Parents and Children: ALSPAC mothers cohort. International journal of epidemiology 2013;42(1):97-110. doi: 10.1093/ije/dys066 [published Online First: 2012/04/18]

32. Sauerbrei Ra. Multivariable Model Building, 2008.

33. Hedrick PW. What is the evidence for heterozygote advantage selection? Trends Ecol Evol 2012;27(12):698-704. doi: 10.1016/j.tree.2012.08.012 [published Online First: 2012/09/15]

34. Kristiansen $M$, Graversen $\mathrm{JH}$, Jacobsen $\mathrm{C}$, et al. Identification of the haemoglobin scavenger receptor. Nature 2001;409(6817):198-201. doi: 10.1038/35051594 [published Online First: 2001/02/24]

35. Lipiski M, Deuel JW, Baek JH, et al. Human Hp1-1 and Hp2-2 phenotype-specific haptoglobin therapeutics are both effective in vitro and in guinea pigs to attenuate hemoglobin toxicity. Antioxidants \& redox signaling 2013;19(14):1619-33. doi: 10.1089/ars.2012.5089 [published Online First: 2013/02/20]

36. Landis RC, Philippidis P, Domin J, et al. Haptoglobin Genotype-Dependent AntiInflammatory Signaling in CD163(+) Macrophages. Int J Inflam 2013;2013:980327. doi: 10.1155/2013/980327 [published Online First: 2013/05/28]

37. Venkatasubramanian C, Mlynash M, Finley-Caulfield A, et al. Natural history of perihematomal edema after intracerebral hemorrhage measured by serial magnetic resonance imaging. Stroke; a journal of cerebral circulation 2011;42(1):73-80. doi: 10.1161/STROKEAHA.110.590646 [published Online First: 2010/12/18]

38. Wu TY, Sharma G, Strbian D, et al. Natural History of Perihematomal Edema and Impact on Outcome After Intracerebral Hemorrhage. Stroke; a journal of cerebral circulation 2017;48(4):873-79. doi: 10.1161/STROKEAHA.116.014416 [published Online First: 2017/03/10]

39. Rodriguez-Luna D, Muchada M, Pineiro S, et al. Potential blood pressure thresholds and outcome in acute intracerebral hemorrhage. European neurology 2014;72(3-4):203-8. doi: 10.1159/000362269

40. Zhao X, Song S, Sun G, et al. Neuroprotective role of haptoglobin after intracerebral hemorrhage. The Journal of neuroscience : the official journal of the Society for Neuroscience 2009;29(50):15819-27. doi: 10.1523/JNEUROSCI.3776-09.2009 


\section{FIGURES}

Figure 1. Patient selection flow diagram

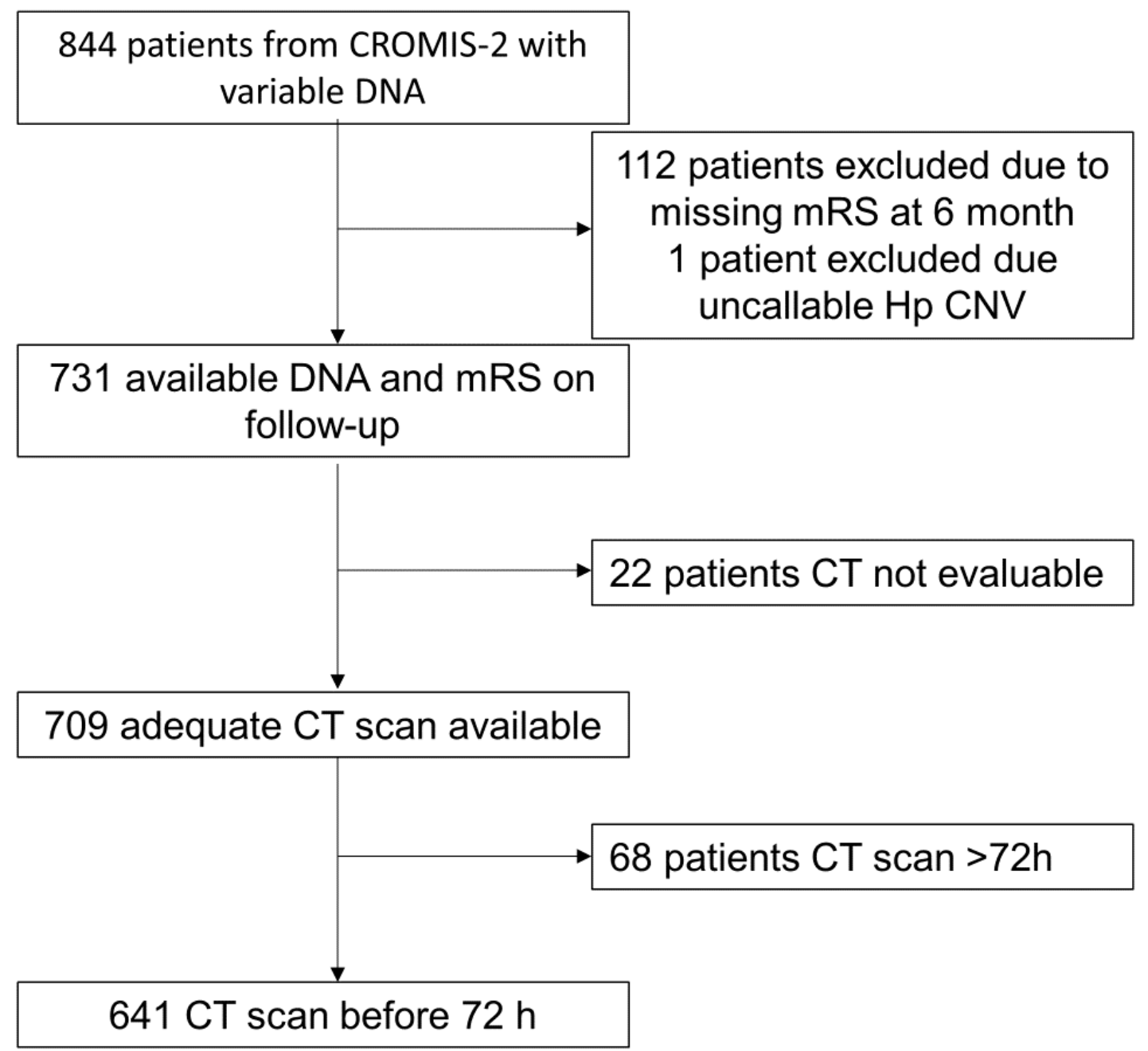


Figure 2. A) Differences in OED in Haptoglobin genotype and SNP, B) Differences in ICH volume in Haptoglobin genotype and SNP

OED volume and genotype and SNP
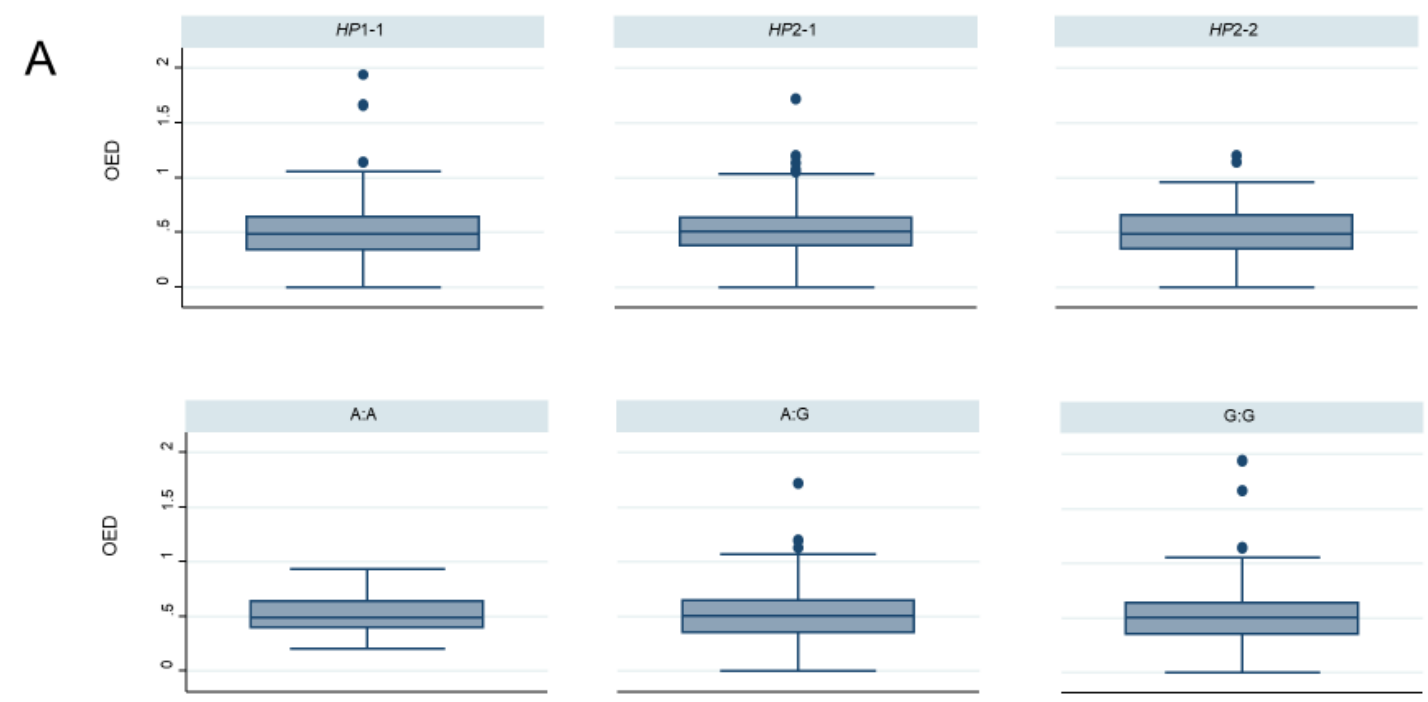

$\mathrm{ICH}$ volume and genotype and SNP
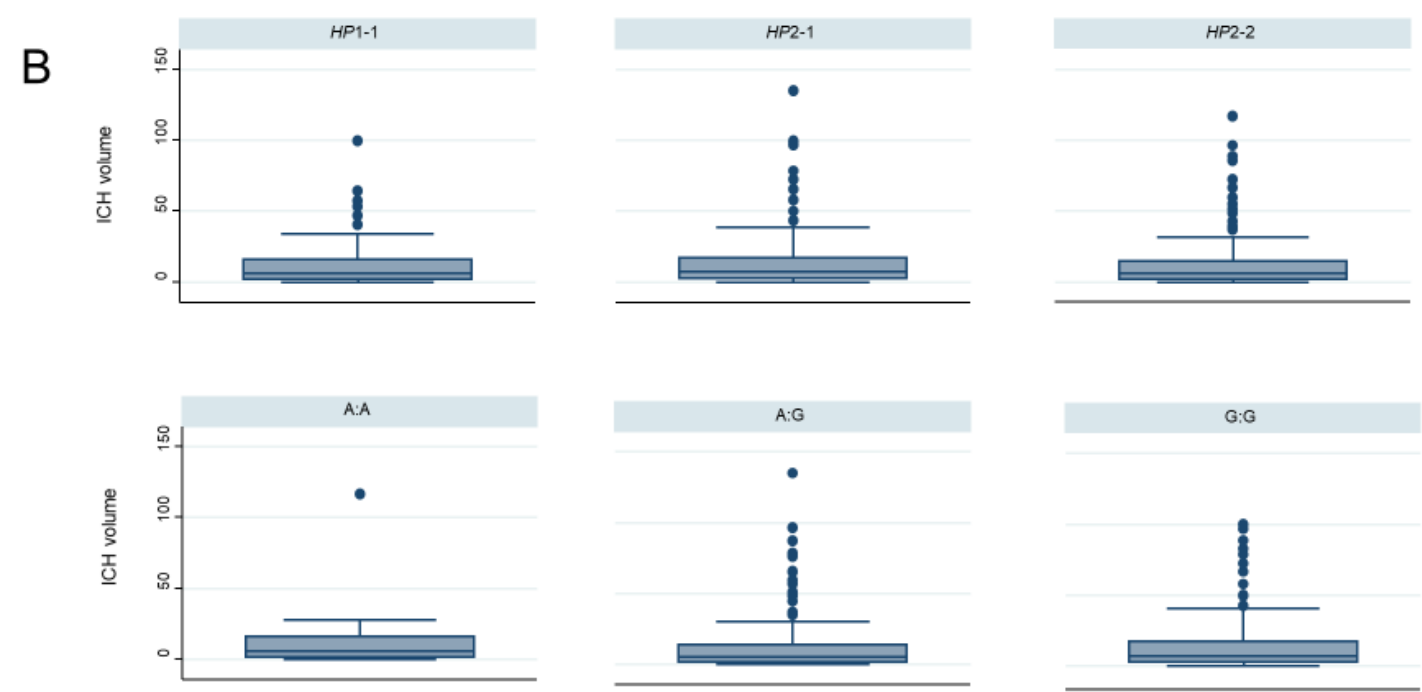
Supplementary Table 1: different in HP CNV and rs2000999 genotype CROMIS versus ALSPAC, genotype frequency

\begin{tabular}{|c|c|c|c|c|}
\hline & Hp1-1 & Hp2-1 & Hp2-2 & Total \\
\hline \multirow{2}{*}{ CROMIS } & 151 & 413 & 279 & 843 \\
& $17.9 \%$ & $49 \%$ & $33.1 \%$ & $100 \%$ \\
\hline \multirow{2}{*}{ ALSPAC } & 137 & 418 & 372 & 927 \\
& $14.8 \%$ & $45.1 \%$ & $40.1 \%$ & $100 \%$ \\
\hline \multirow{2}{*}{ CROMIS } & rs2000999 AA & rs2000999 AG & rs2000999 GG & \\
& 29 & 267 & 523 & 843 \\
& $3.5 \%$ & $32.6 \%$ & $63.9 \%$ & $100 \%$ \\
\hline \multirow{2}{*}{ ALSPAC } & 34 & 229 & 485 & 748 \\
& $4.6 \%$ & $30.6 \%$ & $64.8 \%$ & $100 \%$ \\
\hline
\end{tabular}


Supplementary Table 2: regression of CROMIS versus ALSPAC with regards to rs2000999 genotype and regression of CROMIS versus ALSPAC with regards to HP CNV

\begin{tabular}{|l|l|l|l|}
\hline & OR (95\%CI) & SE & p \\
\hline Hp 1-1 reference & & & 0.007 \\
\hline Hp 2-1 & $0.9(0.69-1.72)$ & 0.12 & \\
\hline Hp 2-2 & $0.68(0.52-0.9)$ & 0.1 & \\
\hline & OR (95\%CI) & SE & p \\
\hline A:A reference & & & 0.47 \\
\hline A:G & $1.37(0.81-2.31)$ & 0.37 & \\
\hline G:G & $1.26(0.76-2.11)$ & 0.33 & \\
\hline
\end{tabular}


Supplementary Table 3: Univariable analysis for functional outcome and death

\begin{tabular}{|c|c|c|c|c|c|c|}
\hline \multirow[t]{2}{*}{ Variable } & \multicolumn{3}{|c|}{ Unfavourable outcome } & \multicolumn{3}{|c|}{ Death } \\
\hline & OR & $95 \% \mathrm{CI}$ & p-value & OR & $95 \% \mathrm{CI}$ & p-value \\
\hline Age, years & 1.05 & $1.03-1.06$ & $<0.001$ & 1.1 & $1.07-1.13$ & $<0.001$ \\
\hline Female Sex & 2.3 & $1.69-3.14$ & $<0.001$ & 1.69 & $1.13-2.53$ & 0.01 \\
\hline Ethnicity & & & 0.71 & & & \\
\hline - White & Reference & & & Reference & & 0.89 \\
\hline - Asian & 1.3 & $0.66-2.58$ & & 0.74 & $0.22-2.53$ & \\
\hline - Black & 1.16 & $0.5-2.66$ & & 0.99 & $0.4-2.43$ & \\
\hline Smoker & 0.83 & $0.61-1.12$ & 0.21 & 1.2 & $0.79-1.8$ & 0.39 \\
\hline Hypertension & 1.79 & $1.31-2.45$ & $<0.001$ & 1.23 & $0.79-1.9$ & 0.36 \\
\hline $\begin{array}{l}\text { SBP on admission, } \\
\mathrm{mmHg}\end{array}$ & 1 & $\begin{array}{l}0.99- \\
1.002\end{array}$ & 0.33 & 0.99 & $0.99-1$ & 0.15 \\
\hline $\mathrm{DM}$ & 1.48 & $0.98-2.26$ & 0.06 & 1.5 & $0.91-2.48$ & 0.11 \\
\hline $\begin{array}{l}\text { Anti-hypertensive } \\
\text { medication }\end{array}$ & 1.45 & $1.07-1.97$ & 0.02 & 1.76 & $1.17-2.65$ & 0.01 \\
\hline Anticoagulation & 1.41 & $1.03-1.94$ & 0.03 & 1.78 & $1.18-2.68$ & 0.006 \\
\hline Antiplatelets & 1.33 & $0.93-1.89$ & 0.11 & 1.04 & $0.65-1.66$ & 0.86 \\
\hline Hypercholesterolaemia & 1.57 & $1.15-2.14$ & 0.004 & 1.09 & $0.72-1.66$ & 0.67 \\
\hline GCS admission & & & & & & \\
\hline - GCS 3-8 & 4.41 & $\begin{array}{l}1.52- \\
12.78\end{array}$ & 0.006 & 5.51 & $2.6-11.64$ & $<0.001$ \\
\hline - GCS 9-15 & Reference & & & Reference & & \\
\hline ICH location & & & 0.63 & & & \\
\hline - Cerebellar & Reference & & & Reference & & 0.1 \\
\hline - Brainstem & 1.35 & $0.4-4.55$ & & 0.34 & $0.04-3.08$ & \\
\hline - Deep & 0.77 & $0.39-1.52$ & & 0.82 & $0.33-2.07$ & \\
\hline - Lobar & 0.84 & $0.42-1.68$ & & 1.36 & $0.54-3.39$ & \\
\hline crICH volume, mL & 1.61 & $1.35-1.93$ & $<0.001$ & 1.88 & $1.52-2.34$ & $<0.001$ \\
\hline IV extension & 2.38 & $1.64-3.45$ & $<0.001$ & 2.62 & $1.72-4$ & $<0.001$ \\
\hline crPHO volume, $\mathrm{mL}$ & 1.39 & $1.17-1.66$ & $<0.001$ & 1.82 & $1.44-2.29$ & $<0.001$ \\
\hline OED, $\mathrm{cm}$ & 1.32 & $0.68-2.54$ & 0.41 & 2.76 & $1.23-6.17$ & 0.01 \\
\hline Neurosurgery & 2.99 & $1-8.94$ & 0.05 & 1.23 & $0.41-3.72$ & 0.71 \\
\hline HP CNV & & & 0.63 & & & 0.15 \\
\hline$-1-1$ & Reference & & & Reference & & \\
\hline$-2-1$ & 0.82 & $0.54-1.24$ & & 0.65 & $0.37-1.12$ & \\
\hline$-2-2$ & 0.84 & $0.54-1.3$ & & 0.96 & $0.55-1.65$ & \\
\hline HP CNV 1 vs all 2 & 0.83 & $0.56-1.22$ & 0.34 & 1.29 & $0.85-1.95$ & 0.23 \\
\hline HP CNV all 1 vs 2 & 0.93 & $0.71-1.33$ & 0.86 & 0.77 & $0.47-1.27$ & 0.31 \\
\hline HP SNP & & & 0.95 & & & 0.49 \\
\hline$-\mathrm{A}: \mathrm{A}$ & Reference & & & Reference & & \\
\hline - A:G & 0.88 & $0.38-2$ & & 0.91 & $0.32-2.54$ & \\
\hline$-G: G$ & 0.9 & $0.4-2.02$ & & 0.71 & $0.26-1.96$ & \\
\hline
\end{tabular}

$\mathrm{Cm}$ = centimetre; $\mathrm{CNV}=$ copy number variation; $\mathrm{crICH}$ = cube root intracerebral haemorrhage; $\mathrm{crPHO}=$ cube root perihaematomal oedema; DM = diabetes mellitus; GCS = Glasgow Coma Scale; HP = Haptoglobin; ICH = intracerebral haemorrhage; IV = intraventricular; $\mathrm{ml}=$ millilitre; $\mathrm{mmHg}$ = millimetre of mercury; OED = oedema extension distance; OR = Odds Ratio; SNP = single nucleotide polymorphism; y = year 
Supplementary Table 4, Factors associated with 6 months mortality after ICH in an adjusted multivariable logistic regression model, HP CNV dichotomized

\begin{tabular}{|c|c|c|c|}
\hline & OR & $95 \% \mathrm{CI}$ & $P$ value \\
\hline Age (years) & 1.11 & $1.07-1.14$ & $<0.001$ \\
\hline Female Sex & 1.15 & $0.69-1.94$ & 0.6 \\
\hline Hypertension & 0.98 & $0.56-1.72$ & 0.95 \\
\hline Diabetes mellitus & 1.33 & $0.65-2.69$ & 0.43 \\
\hline Oral anticoagulation & 1.27 & $0.75-2.13$ & 0.37 \\
\hline $\begin{array}{l}\text { GCS on admission (binary) } \\
\text { - GCS 3-8 } \\
\text { - GCS 9-15 (reference) }\end{array}$ & 4.37 & $1.39-13.73$ & 0.01 \\
\hline $\begin{array}{l}\text { ICH location } \\
\text { - Cerebellar (reference) }\end{array}$ & & & 0.39 \\
\hline - Brainstem & $\begin{array}{c}\text { No } \\
\text { observations }\end{array}$ & & \\
\hline $\begin{array}{l}\text { - Deep } \\
\text { - Lobar }\end{array}$ & $\begin{array}{l}0.94 \\
0.62\end{array}$ & $\begin{array}{l}0.32-2.8 \\
0.2-1.93\end{array}$ & \\
\hline Cr ICH volume (mL) & 1.99 & $1.45-2.74$ & $<0.001$ \\
\hline $\mathrm{OED}(\mathrm{cm})$ & 3 & $1.07-8.39$ & 0.04 \\
\hline IV extension & 1.57 & $0.9-2.75$ & 0.11 \\
\hline \multicolumn{4}{|l|}{ HP CNV } \\
\hline - HP1 allele (HP1-1/2-1 vs HP2-2) & 0.55 & $0.31-0.95$ & 0.03 \\
\hline $\begin{array}{l}\text { Rs2000999 } \\
\text { - A:A (reference) } \\
\text { - A:G } \\
\text { - G:G }\end{array}$ & $\begin{array}{l}0.61 \\
0.61\end{array}$ & $\begin{array}{c}0.16-2.4 \\
0.16-2.41\end{array}$ & 0.77 \\
\hline
\end{tabular}

$\mathrm{Cm}$ = centimetre; $\mathrm{CNV}=$ copy number variation, $\mathrm{Cr}$ = cube root; $\mathrm{CT}=$ computed tomography; $\mathrm{GCS}=$ Glasgow Coma Scale; HP = Haptoglobin; ICH = intracerebral haemorrhage; IV = intraventricular; OAC = oral anticoagulation; SBP = systolic blood pressure 


\section{Supplementary Table 5: Univariable analysis for ICH and OED volume}

\begin{tabular}{|c|c|c|c|c|c|c|}
\hline \multirow[t]{2}{*}{ Variable } & \multicolumn{3}{|c|}{ ICH volume } & \multicolumn{3}{|c|}{ OED volume } \\
\hline & Coefficient & $95 \% \mathrm{CI}$ & p-value & Coefficient & $95 \% \mathrm{CI}$ & p-value \\
\hline Time event to CT & & & 0.5 & & & 0.13 \\
\hline - Day 1 & Reference & & & & & \\
\hline - Day 2 & 0.18 & $-0.12-0.48$ & & 0.07 & $0.001-0.15$ & \\
\hline - Day 3 & -0.17 & $-0.61-0.27$ & & 0.03 & $-0.09-0.15$ & \\
\hline Age, years & 0.004 & $-0.002-0.01$ & 0.2 & 0.001 & $-0.001-0.002$ & 0.25 \\
\hline Female Sex & 0.05 & $-0.1-0.2$ & 0.52 & 0.02 & $-0.01-0.06$ & 0.19 \\
\hline Smoker & -0.03 & $-0.11-0.04$ & 0.39 & -0.008 & $-0.03-0.01$ & 0.4 \\
\hline Hypertension & 0.06 & $-0.1-0.22$ & 0.45 & -0.005 & $-0.04-0.03$ & 0.77 \\
\hline $\begin{array}{l}\text { SBP on admission, } \\
\mathrm{mmHg}\end{array}$ & -0.002 & $-0.004-0.0005$ & 0.12 & 0.0003 & $\begin{array}{l}-0.0005- \\
0.0007\end{array}$ & 0.66 \\
\hline $\begin{array}{l}\text { Anti-hypertensive } \\
\text { medication }\end{array}$ & 0.08 & $-0.07-0.23$ & 0.29 & -0.07 & $-0.1-0.03$ & $<0.001$ \\
\hline Anticoagulation & 0.09 & $-0.06-0.25$ & 0.25 & -0.05 & $-0.09-(-0.01)$ & 0.01 \\
\hline Antiplatelets & 0.01 & $-0.16-0.18$ & 0.89 & -0.03 & $-0.07-0.02$ & 0.21 \\
\hline High cholesterol & 0.16 & $0.01-0.32$ & 0.03 & -0.006 & $-0.04-0.03$ & 0.74 \\
\hline $\begin{array}{l}\text { Platelet level, } \\
\text { x10\%/liter }\end{array}$ & 0.001 & $-0.0002-0.002$ & 0.13 & 0.0003 & $0.0001-0.001$ & 0.002 \\
\hline ICH location & & & $<0.001$ & & & $<0.001$ \\
\hline - Cerebellar & Reference & & & & & \\
\hline - Brainstem & -0.86 & $-1.34-(-0.37)$ & & -0.09 & $-0.21-0.04$ & \\
\hline - Deep & -0.19 & $-0.49-0.11$ & & 0.15 & $0.07-0.23$ & \\
\hline - Lobar & 0.67 & $0.37-0.97$ & & 0.23 & $0.15-0.31$ & \\
\hline IV extension & 0.46 & $0.29-0.62$ & $<0.001$ & -0.03 & $-0.07-0.01$ & 0.16 \\
\hline Neurosurgery & 0.62 & $0.15-1.09$ & 0.01 & -0.003 & $-0.12-0.12$ & 0.95 \\
\hline HP CNV & & & 0.35 & & & 0.84 \\
\hline$-1-1$ & Reference & & & & & \\
\hline$-2-1$ & 0.11 & $-0.09-0.34$ & & 0.004 & $-0.05-0.06$ & \\
\hline$-2-2$ & 0.02 & $-0.2-0.24$ & & -0.01 & $-0.06-0.05$ & \\
\hline HP CNV all 1 vs $2-2$ & -0.07 & $-0.22-0.09$ & 0.39 & -0.01 & $-0.05-0.03$ & 0.57 \\
\hline HP CNV all 2 vs $1-1$ & 0.08 & $-0.12-0.28$ & 0.42 & -0.001 & $-0.05-0.05$ & 0.96 \\
\hline HP SNP & & & 0.82 & & & 0.98 \\
\hline - A:A & Reference & & & & & \\
\hline$-A: G$ & 0.03 & $-0.39-0.45$ & & -0.002 & $-0.1-0.1$ & \\
\hline - G:G & 0.08 & $-0.33-0.49$ & & -0.005 & $-0.11-0.1$ & \\
\hline
\end{tabular}

$\mathrm{CNV}=$ copy number variation; $\mathrm{HP}=$ Haptoglobin; $\mathrm{ICH}=$ intracerebral haemorrhage; $\mathrm{IV}=$ intraventricular; OED = oedema extension distance; SBP = systolic blood pressure; SNP = single nucleotide polymorphism 
Supplementary Figure 1. A) Time to death in days by HP CNV overall cohort, B) Time to death in days by rs2000999 overall cohort, C) Time to death in day by HP CNV subgroup >80 years $<12.2 \mathrm{~mL}$ ICH volume, D) Time to death in day by rs2000999 subgroup $>80$ years $<12.2 \mathrm{~mL}$ ICH volume

A

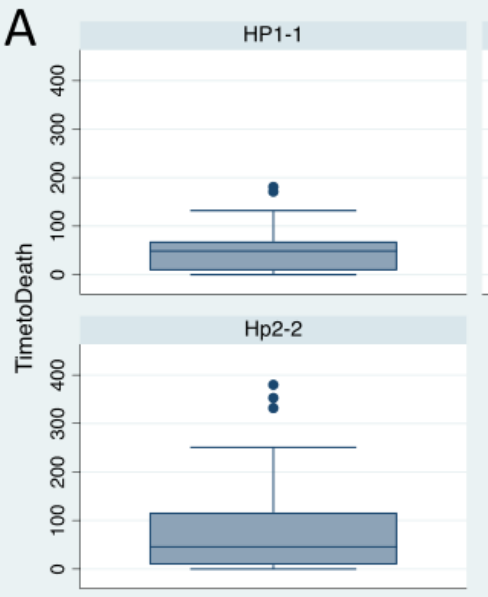

Graphs by HP genotype

C

C HP1-1

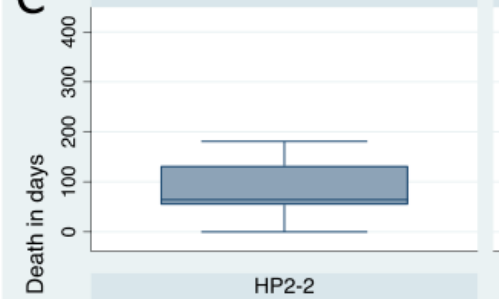

올

\&

Graphs by HP genotype
B
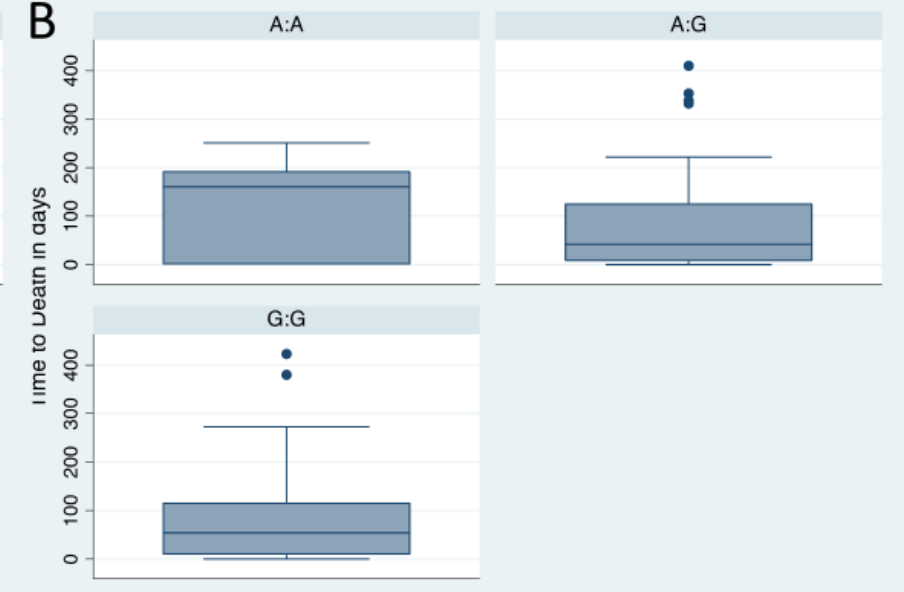

Graphs by HP SNP rs2000999

$\mathrm{D}$

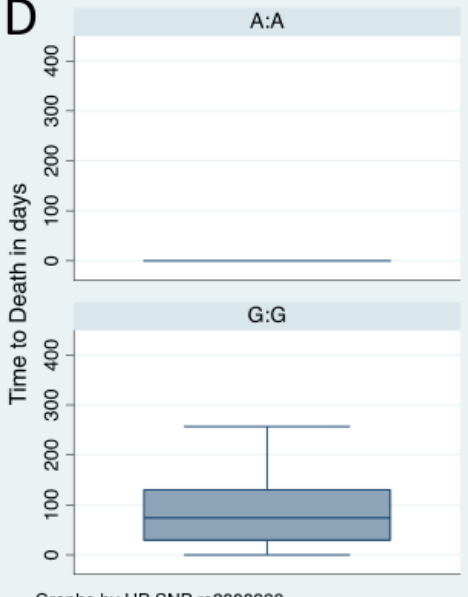

Graphs by HP SNP rs2000999 